\title{
Energy efficiency in MAC 802.15.4 for wireless sensor networks
}

\begin{abstract}
Recent technological advances in sensors, low power integrated circuits, and wireless communications have enabled the design of low-cost, lightweight, and intelligent physiological sensor nodes. The IEEE 802.15.4 is a new wireless personal area network designed for wireless monitoring and control applications. The fast progress of research on energy efficiency in wireless sensor networks, and the need to compare with the solutions adopted in the standards motivates the need for this work. In the analysis presented, the star network configuration of 802.15.4 standard at $868 \mathrm{MHz}$ is considered for a Zigbee network. In this paper, we analyze the active duration of the superframe and entered the sleep mode status inside this period. It happens when sensors do not have any data to send. The nonpersistent CSMA uses the adaptive backoff exponent. This method helps the network to be reliable under traffic changes due to save the energy consumption. The introduction of sleep state has shown incredible reduction of the power consumption in all network load changes.
\end{abstract}

Keyword: Energy efficiency; IEEE 802 15.4; Media access control; Wireless sensor network; Zigbee 